\title{
Fruit and vegetable consumption assessed by three dietary assessment methods in regard to sex, age, BMI and socio economic status
}

\begin{abstract}
Introduction: A comparison of means of food consumption assessed by three different dietary assessment methods (diet history interviews [DHI], 24h-recalls [24HR] and weighing food records [WR]) used in the German National Nutrition Survey (NVS) II showed higher consumption means in 7 out of 18 food groups for DHI compared to 24HR and WR. Especially for food groups perceived as socially desirable such as fruit and vegetable means were highest for DHI. In the following, it is examined whether differences in fruit and vegetable consumption assessed by three different dietary assessment methods are related to sex, age, body mass index (BMI) or socio economic status (SES).
\end{abstract}

Methods: A subgroup of 677 participants of the NVS II (2005-2007, 14-80 years of age) completed all three dietary assessment methods. DHI covered the food consumption of the past month, 24HR of the previous day and WR two times four days. Body height and weight were measured. SES was defined as an index based on the household income, employment status of the household's principle earner, and education level of the participant. The Multiple Source Method was applied to estimate population distributions of usual intakes based on two 24HR. Confidence intervals were calculated on basis of bootstrapping samples. Differences are considered to be significant if confidence intervals do not overlap.

Results: For vegetable consumption, all subgroups regarding sex (male, female), age (14-18 years, 19-24 years, 25-34 years, 51-64 years, 65 years and older), body mass index $\left(<25 \mathrm{~kg} / \mathrm{m}^{2}, 25-30 \mathrm{~kg} / \mathrm{m}^{2},>30 \mathrm{~kg} / \mathrm{m}^{2}\right)$ and SES $(5$ groups from $1=1$ lower to $5=$ upper SES) showed higher means for DHI compared to 24HR and WR. For fruit consumption, in almost all subgroups higher means for DHI compared to 24HR and WR could be found, except for the age group 19-24 years and the lowest SES group.

Discussion: The results show that higher means in fruit and vegetable consumption assessed by DHI compared to 24HR and WR are independent of sex, age, BMI and SES. A reason why socially desirable foods like fruit or vegetables are stated in higher amounts by DHI may be the enormous cognitive task of participants necessary to estimate quantities and frequencies over the long period of time covered by DHI.

\section{Conflict of Interest}

There is no conflict of interest. 